\title{
Broncholithiasis, Lithoptysis und broncho-ösophageale Fistel
}

\author{
Broncholithiasis, Lithoptysis and Broncho-oesophageal Fistula
}

Autoren

Institute
M. Westhoff ${ }^{1}$, P. Litterst ${ }^{1}$, F. Al-Shahrabani ${ }^{2}$

${ }^{1}$ Klinik für Pneumologie, Hemer

${ }^{2}$ Klinik für Thoraxchirurgie, Lungenklinik Hemer, Hemer eingereicht $\quad 30.7 .2011$ akzeptiert nach Revision 15. 8. 2011

\section{Bibliografie}

DOI http://dx.doi.org/ 10.1055/s-0031-1286633 Online-Publikation: 2.11 .2011 Pneumologie 2012; 66: 74-77 (c) Georg Thieme Verlag KG Stuttgart · New York ISSN 0934-8387

\section{Korrespondenzadresse} Dr. Michael Westhoff Klinik für Pneumologie Lungenklinik Hemer Theo-Funcciusstr. 1 58675 Hemer Michael.Westhoff@lkhemer.de

\section{Zusammenfassung \\ $\nabla$}

Broncho-ösophageale Fisteln bei Broncholithiasis sind extrem selten. Eine vorausgehende Lithoptysis wird nur vereinzelt beschrieben. Berichtet wird über einen 68-jährigen Patienten, der über eine mehr als 3 Jahre bestehende Hustensymptomatik klagte. 5 Monate vor der stationären Aufnahme trat eine Hämoptyse mit begleitendem Abhusten von mehreren gräulichen, bis zu $5 \mathrm{~mm}$ im Durchmesser großen Steinen auf. Bei der Bronchoskopie zeigte sich eine kleine Exkavation an der medialen Wand des linken Hauptbronchus. Im Vergleich zu einem vorausgegangenen CT konnte auf einem aktuellen CT eine kleine Fistel zwischen Ösophagus und linkem Hauptbronchus sowie eine nicht mehr vorhandene Kalzifikation in diesem Bereich nachgewiesen werden. Der Patient wurde linksseitig thorakomiert. Es erfolgte die Exzision der Fistel, Übernähung des Ösophagus und Interposition eines M. latissimus dorsi Lappens. Der Nachweis einer Broncholithwanderung mittels $\mathrm{CT}$ vor und nach der Lithoptyse, die Ausbildung einer linksseitigen Fistel und ihre Darstellung im CT sowie bei der Bronchoskopie wurden in dieser Kombination bislang nicht in der Literatur berichtet.

\section{Einleitung}

$\nabla$

Ein persistierender Husten ist ein häufiges klinisches Symptom in der Pneumologie. Wenn dieses mit wiederkehrender Aspiration, mediastinaler Lymphknotenverkalkung und Abhusten von „Steinen“ assoziiert ist, müssen auch seltene Ursachen in Erwägung gezogen werden.

Nachfolgend wird ein ungewöhnlicher Fall eines Patienten mit mediastinalen Lymphknotenverkalkungen vorgestellt, deren Wanderung in einer Broncholithiasis mit Lithoptysis und symptomatischer broncho-ösophagealer Fistel resultierte.

\section{Abstract \\ $\nabla$}

Broncho-oesophageal fistulas in broncholithiasis are extremely rare. Preceding lithoptysis is only seen infrequently. We report on a 68 -year-old patient who complained of cough for more than 3 years. 5 months prior to admission he had hemoptysis and expectorated several greyish stones of up to $5 \mathrm{~mm}$ diameter. Endoscopy revealed a small excavation at the medial wall of the left main bronchus. Compared to a former CT, an actual CT scan of the thorax showed a small fistula between the oesophagus and the left main bronchus and revealed a missing calcification at this site. The patient underwent a left-sided thoracotomy with excision of the fistula, suture of the oesophagus and interponation of a flap of the M. latissimus dorsi. The demonstration of broncholith migration with CT scans before and after lithoptysis, the development of a left-sided fistula and its demonstration in the CT scan as well as endoscopically have not been reported in this combination before.

Der Verlauf konnte klinisch und radiologisch dokumentiert werden.

\section{Kasuistik \\ $\nabla$ \\ Anamnese}

Der 68-jährige Patient beklagte seit etwa 3 Jahren einen persistierenden Husten. Deshalb erfolgte im Juni 2008 eine Diagnostik in einer auswärtigen Klinik. Das CT zeigte Verkalkungen der mediastinalen Lymphknoten ( $\boldsymbol{A} \mathbf{A b b} . \mathbf{1} \mathbf{a}$ u.b). In einer polypoiden Struktur des rechten Hauptbronchus fanden sich epitheloidzellartige Granulome und 


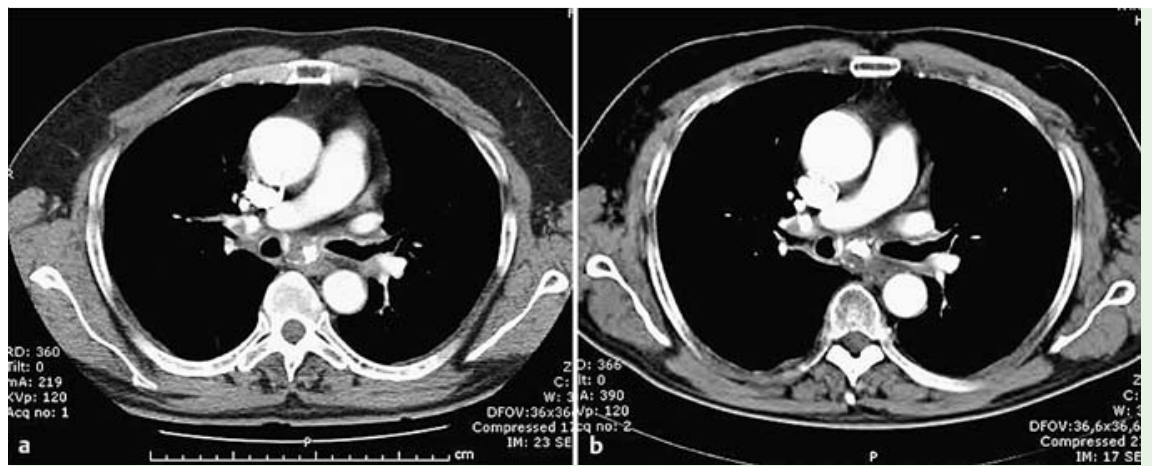

Abb. 1 a, b CT-Thorax 6/2008. Nachweis von Verkalkungen in mediastinalen Lymphknoten.

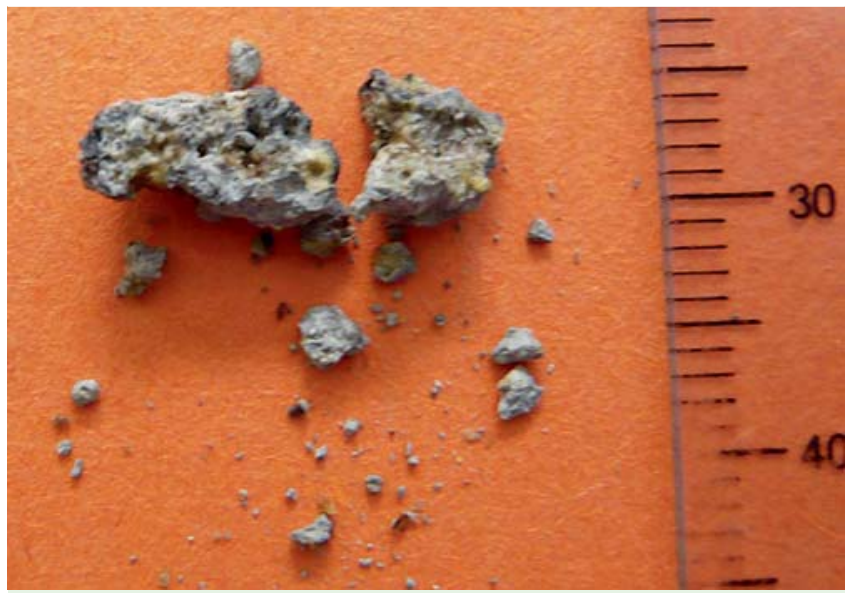

Abb. 2 Abgehusteter Broncholith.

Nekrosen. Im Verlauf persistierte der Husten nach Trinken von Flüssigkeiten. Im Dezember 2008 hustete der Patient blutiges Sekret und mehrere bis zu $5 \mathrm{~mm}$ große gräuliche Steine ab ( $\bullet$ Abb.2), die er zur stationären Aufnahme im Mai 2009 mitbrachte.

\section{Klinischer Befund}

Der klinische Untersuchungsbefund war regelrecht. Über den Lungen konnte reines Vesikuläratmen auskultiert werden.

\section{Labor}

CRP und klinisches Routinelabor (Blutbild, Leber- und Nierenwerte) boten keine Auffälligkeiten.

\section{Endoskopie (Laryngoskopie und Bronchoskopie)}

Es bestand ein unauffälliger Larynxbefund mit normaler Stimmwandbeweglichkeit. In der medialen Wand des linken Hauptbronchus bestand eine winzig kleine Exkavation ( $\bullet$ Abb.3). Aufgrund der Angabe von Hustenattacken nach Trinken von Flüssigkeiten erfolgte eine Laryngoskopie beim Trinken von gefärbter Flüssigkeit. Diese wurde nach dem ersten Schluckakt abgehustet.

\section{Computertomografie}

Das Kontroll-CT zeigte im Vergleich zum Vor-CT aus dem Jahr 2008 eine kleine Fistel zwischen Ösophagus und linkem Hauptbronchus ( $\bullet$ Abb.4au.b). Gegenüber dem Vor-CT fehlte in der LK-Station 7 ein Kalkfragment ( Abb.5au.b).

\section{Therapie}

Bei endoskopisch und radiologisch nachgewiesener und klinisch eindeutig symptomatischer broncho-ösophagealer Fistel bestand die Indikation zur operativen Fistelsanierung. Therapeutisch erfolgte die Thorakotomie links mit Fistelexzision, Ösophagusübernähung und Latissimus-Interponationsplastik. Im Verlauf war der Patient asymptomatisch. TB-Kulturen von Sekret und intraoperativen Abstrichen waren negativ.

\section{Diskussion}

\section{$\nabla$}

Die in der Kasuistik beschriebene klinische Symptomatik ist pathognomonisch für eine Broncholithiasis mit begleitender aerodigestiver Fistel. Abgesehen vom klinischen Verlauf stellen der Nachweis einer Broncholithwanderung mittels CT vor und nach der Lithoptyse, die Ausbildung einer linksseitigen Fistel und ihre Darstellung im CT sowie bei der Bronchoskopie eine Besonderheit dar, die in dieser Kombination bislang nicht in der Literatur berichtet wurde.

Eine klinische Symptomatik mit wiederholtem Auftreten von Husten nach Trinken von Flüssigkeiten ist primär verdächtig auf rezidivierende Aspirationen. Die Differenzialdiagnostik umfasst neurologische Ursachen wie eine ALS mit bulbärer Symptomatik sowie Fistelbildungen zwischen dem Ösophagus und Tracheobronchialsystem. Diese sind häufig Folgen maligner Tumoren, insbesondere des Ösophagus, wobei die Diagnose und Symptomatik der Tumorerkrankung in der Regel schon längere Zeit vor dem Auftreten der Aspirationssymptome bekannt sind und oft im Vordergrund stehen.

Wiederholte Aspirationen ohne begleitende klinische Symptome einer neurologischen oder Tumorerkrankung müssen auch an das Vorliegen benigner aerodigestiver Fisteln denken lassen. Diese werden allerdings nur in seltensten Fällen beobachtet. So berichten Mangi et al. [1] über 13 Fälle in einem Zeitraum von 41 Jahren; in diesem Kollektiv war jedoch keine Fistel mit einer Broncholithiasis vergesellschaftet.

Klinische Symptome einer wiederkehrenden Aspiration erfordern neben der Bildgebung in Form eines Röntgen- bzw. CT-Thorax eine endoskopische Abklärung des Tracheobronchialsystems und eine (video-) endoskopische Untersuchung des Schluckaktes. Hierbei kann zwischen den verschiedenen Formen der klassischen Aspiration mit Übertritt von Farblösung in den Kehlkopf und die Trachea sowie dem Abhusten gefärbter Lösung ohne vorherige laryngeale Aspiration differenziert werden. Letztere ist wie im dargestellten Fall typisch für das Vorliegen einer tracheooder broncho-ösophagealen Fistel, ohne zunächst Aussagen über deren Genese zu erlauben. Der Verdacht auf eine aerodigestive Fistel erfordert eine sorgfältige endoskopische Untersuchung, 

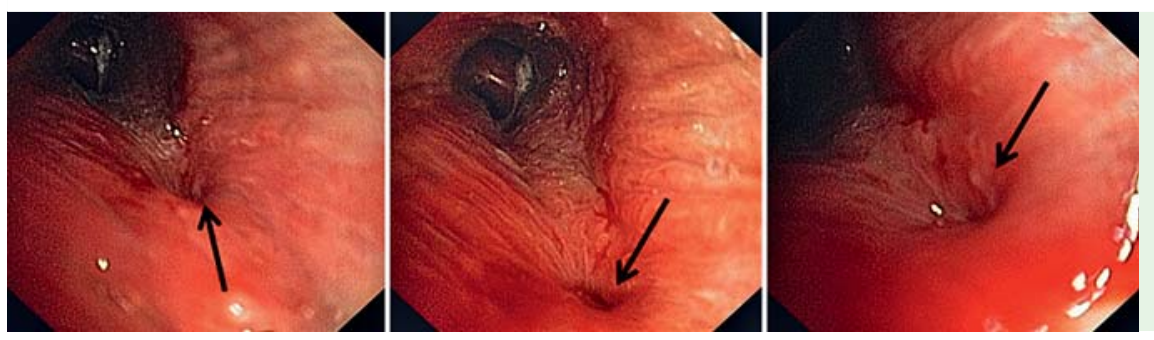

Abb.3 Fistelöffnung ( $\uparrow)$ im linken Hauptbronchus.
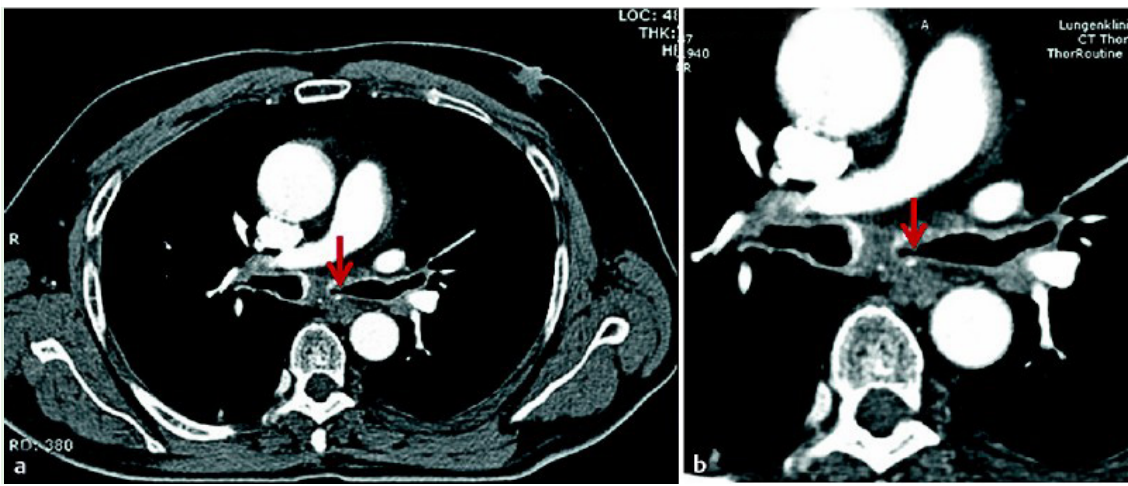

Abb. 4a,b CT-Thorax 5/2009. Fistel zwischen Ösophagus und linkem Hauptbronchus ( $\downarrow$ ).
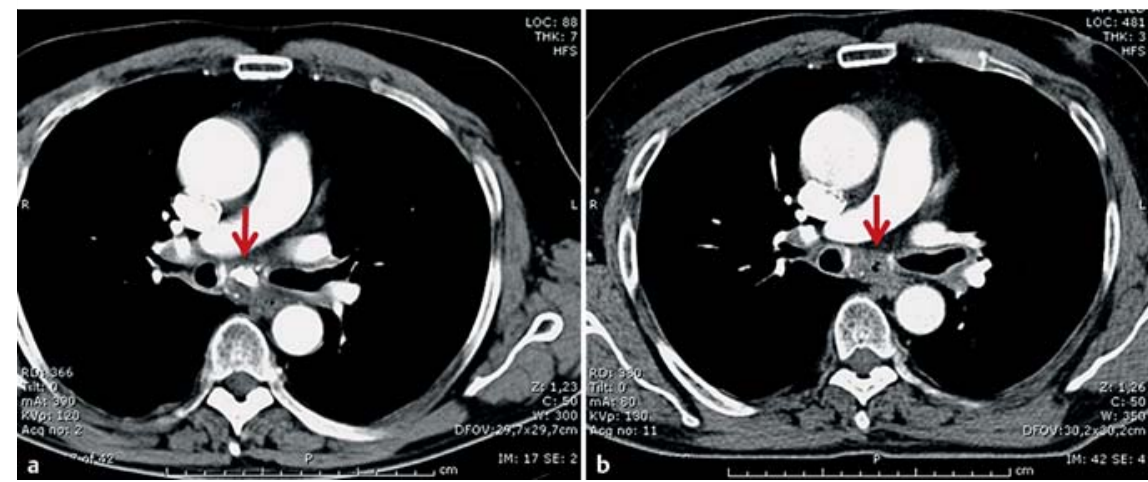

Abb. 5 a CT-Thorax 6/2008 und b CT-Thorax 5/2009. Im Verlauf nicht mehr vorhandenes Kalkfragment medial des linken Hauptbronchus ( $\downarrow$ ).

um auch kleinste Fistelöffnungen im Tracheobronchialsystem zu entdecken. Allerdings ist es nicht ungewöhnlich, auch Fistelöffnungen zu übersehen. Der vorliegende Fall unterstreicht deshalb in besonderem Maße, dass gegebenenfalls wiederholte endoskopische Untersuchungen einschließlich Ösophagoskopie und Bronchoskopie im Rahmen der Diagnostik erforderlich sind.

Der Nachweis kalzifizierter Strukturen im Bronchialsystem - sei es endoskopisch oder radiologisch - ist ein Indiz für eine zugrundeliegende Broncholithiasis, ohne jedoch weitere Rückschlüsse über die zugrundeliegende Ursache der Lithiasis zu erlauben. Die Broncholithiasis ist zunächst nur durch das Auftreten von kalzifiziertem Material innerhalb des Bronchiallumens gekennzeichnet und stellt einen extrem seltenen Befund dar. Olson et al. [2] berichten von 95 Fällen aus der Mayo Clinic in einem Zeitraum von 40 Jahren. Die Broncholithiasis ist im Wesentlichen assoziiert mit Infektionen wie einer Tuberkulose, Histoplasmose, Aktinomykose oder Nocardiose, aber auch bei Silikosen beobachtet worden [2-13]. Im aktuellen Fall ergaben sich keine eindeutigen Beweise für eine derartige Genese, wenngleich vom Alter und der Herkunft des Patienten postspezifische Verkalkungen am wahrscheinlichsten erscheinen.

Die polypoide Struktur, die bei der auswärtigen Bronchoskopie vor dem Abhusten der Broncholithen gesehen wurde, muss als granulomatöse Reaktion auf die Kalzifikationen und eine beginnende Wanderungstendenz gedeutet werden. Allerdings trat eine weitere Wanderung der Kalzifikationen in dem Bereich, in dem die polypoiden Strukturen beobachtet wurden, nicht auf, sodass zu diskutieren ist, dass die überschießende granulomatöse Veränderung durchaus einen weiteren Wanderungsprozess verhindert hat. Inwieweit die initialen Symptome mit Husten vor dem Auftreten der Lithoptysis möglicherweise schon von einer kleinen Fistel zwischen dem linken Hauptbronchus und dem Ösophagus resultierten, bleibt spekulativ.

Das Auftreten einer aerodigestiven Fistel im Zusammenhang mit einer Broncholithiasis ist nur vereinzelt beobachtet worden. Cerfolio et al. [14] berichten von nur 2 Fisteln bei 50 Patienten mit Broncholithiasis. Eine retrospektive Analyse über 38 Jahre aus der Mayo Klinik [15] konnte 9 Patienten mit broncho-ösophagealer Fistel ausmachen: davon waren 7 Fisteln rechtsseitig lokalisiert, entsprechend der prädominierenden Lokalisation benigner broncho-ösophagealer Fisteln anderer Ursache [2,16]. Insofern stellt die im dargestellten Fall beobachtete linksseitige Fistelöffnung eine ungewöhnliche Lokalisation dar.

Das Abhusten von „Steinen“, also kalkhaltigem Material, wird als „lithoptysis“ oder „phtisis calculosa“ $[3,17]$ bezeichnet und ist pathognomonisch für eine zugrundeliegende Broncholithiasis und Wanderung des Broncholithen. In der Literatur finden sich nur einzelne Fallberichte über benigne broncho-ösophageale Fisteln, die durch eine Broncholithiasis verursacht und durch eine Lithoptysis symptomatisch wurden [3,17-19]. Wie im darge- 
stellten Fall war die Lithoptysis immer mit einer Hämoptyse vergesellschaftet.

Der Nachweis einer broncho-ösophagealen Fistel im CT ist ungewöhnlich und kann primär nicht erwartet werden. Auch im dargestellten Fall konnte im ersten CT-Thorax keine Fistel entdeckt werden. Erst nach der Expektoration von multiplen Fragmenten eines großen Broncholithen stellte sich im Kontroll-CT die linksseitige broncho-ösophageale Fistel bei gleichzeitigem Fehlen einer Kalzifikation eines mediastinalen Lymphknotens dar. Dies unterstreicht, dass auf Veränderungen im Erscheinungsbild und der Größe bekannter mediastinaler oder hilärer Kalzifikationen geachtet werden muss, da sie als Hinweis auf eine Broncholithiasis und ihre möglichen Komplikationen dienen können, insbesondere wenn typische klinische Symptome wie rezidivierender Husten, gegebenenfalls sogar mit Expektoration von kalkhaltigem Material, koexistieren.

Zur Vermeidung weiterer Aspirationen ist ein definitiver Verschluss aerodigestiver Fisteln erforderlich. Therapie der Wahl ist deshalb die operative Resektion der Fistel über eine Thorakotomie mit Übernähung von Trachea, Bronchus und Ösophagus sowie gleichzeitiger plastischer Deckung mittels eines Muskelinterponats $[1,11,16,17]$. In Einzelfällen mit Inoperabilität können endoskopische Verfahren mit Fistelverklebung allein oder mit zusätzlichem Clipping [20,21] zum Einsatz kommen.

Zusammenfassend ist der dargestellte Fall bezüglich der Symptomatik rezidivierender Aspirationen sowie des klinischen Verlaufs einer Broncholithiasis mit Migration, Lithoptysis und Fistelausbildung exemplarisch.

\section{Interessenkonflikt}

$\nabla$

Die Autoren geben an, dass kein Interessenkonflikt besteht.

\section{Literatur}

1 Mangi AA, Gaissert HA, Wright CD et al. Benign broncho-oesophageal fistula in the adult. Ann Thorac Surg 2002; 73: 911 - 915

2 Olson EJ, Utz JP, Prakash UB. Therapeutic bronchoscopy in broncholithiasis. AmJ Respir Crit Care Med 1999; 160: 766-770

3 Kidd HM, Christopherson E. Broncholithiasis and broncho-oesophageal fistula. Can Med Assoc J 1951; 64: 142 - 146
4 Seo JB, Song KS, Lee JS et al. Broncholithiasis: review of the causes with radiologic-pathologic correlation. Radiographics 2002; 22: S199-213

5 Antão VC, Pinheiro GA, Jansen JM. Broncholithiasis and lithoptysis associated with silicosis. Eur Respir J 2002; 20: 1057-1059

6 Hammoud ZT, Rose AS, Hage CA et al. Surgical management of pulmonary and mediastinal sequelae of histoplasmosis: a challenging spectrum. Ann Thorac Surg 2009; 88: 399-403

7 Hirschfield LS, Graver LM, Isenberg HD. Broncholithiasis due to Histoplasma capsulatum subsequently infected by actinomycetes. Chest 1989; 96: 218-219

$8 \mathrm{Kim}$ TS, Han J, Koh WJ et al. Endobronchial actinomycosis associated with broncholithiasis: CT findings for nine patients. AJR Am J Roentgenol 2005; 185: 347-353

9 Reddy AJ, Govert JA, Sporn TA et al. Broncholith removal using cryotherapy during flexible bronchoscopy: a case report. Chest 2007; 132: $1661-1663$

10 Samson IM, Rossoff LJ. Chronic lithoptysis with multiple bilateral broncholiths. Chest 1997; 112: 563-565

11 Faber LP, Jensik RJ, Chawla SK et al. The surgical implication of broncholithiasis. J Thorac Cardiovasc Surg 1975; 70: 779-789

12 Weed LA, Andersen HA. Etiology of broncholithiasis. Dis Chest 1960; 37: $270-277$

13 Tsubochi H, Endo S, Suhara Ket al. Endobronchial aspergillosis and actinomycosis associated with broncholithiasis. Eur J Cardiothorac Surg 2007; 31: $1144-1146$

14 Cerfolio RJ, Bryant AS, Maniscalco L. Rigid bronchoscopy and surgical resection for broncholithiasis and calcified mediastinal lymph nodes. J Thorac Cardiovasc Surg 2008; 136: 186-190

15 Ford MA, Mueller PS, Morgenthaler TI. Bronchoesophageal fistula due to broncholithiasis: a case series. Respir Med 2005; 99: 830-835

16 Manac'h D, Riquet M, Plaza de los Reyes $M$ et al. Esophago-bronchial fistula in adults. Ann Chir 1990; 44: 636-641

17 Carvajal Balaguera J, Mallagray Casas S, Martínez Cruz R et al. Bronchoesophageal fistula and broncholithiasis. Arch Bronconeumol 1995; 32: $184-187$

18 Tinney WS, Moersch HJ. Broncholithiasis. Surg Clin North Am 1944; 24: $830-838$

19 Shaaban AM, Mann H, Morrell G et al. A case of broncholithiasis and esophagobronchial fistula. J Thorac Imaging 2007; 22: 259-262

20 DeLuca G, Griffo S, Monaco $M$ et al. Combined endoscopic approach in the treatment of benign broncho-oesophageal fistula. Thorax 2008; 63: $1024-1025$

21 Yellapu RK, Gorthi JR, Kiranmayi Yet al. Endoscopic occlusion of idiopathic benign esophago-bronchial fistula. J Postgrad Med 2010; 56: $284-286$ 